\title{
Situs Inversus Totalis asociado a síndrome de preexcitación ventricular. Reporte de un caso.
}

\author{
Matías Carreño ${ }^{2}$, José Miguel Castellón ${ }^{3}$, Francisca Iglesias ${ }^{1}$, Diego Morales ${ }^{1}$. \\ 1. Médico Cirujano, Médico General de Zona Servicio Metropolitano Suroriente, CESFAM Vista Hermosa, Puente Alto, Santiago. \\ 2. Médico Cirujano, CESFAM San Luis, Peñalolén, Santiago. \\ 3. Médico Cirujano, Magister en Investigación Clínica, Universidad de Barcelona.
}

El situs inversus totalis es la inversión congénita completa de órganos torácicos y abdominales. Se presenta el caso de una paciente de 3 años sin antecedentes médicos previos, a quien en atención primaria, y por un cuadro respiratorio agudo, se evidencia el hallazgo de dextrocardia y burbuja gástrica a derecha en la radiografía de tórax, sospechándose situs inversus totalis. Fue derivada a cardiología infantil donde se confirmó el diagnóstico con un ecocardiograma transtorácico, asociado a un hallazgo de preexcitación ventricular en el electrocardiograma. Una vez resuelto el cuadro respiratorio agudo, la paciente se mantiene controlada de manera periódica en atención primaria y por especialista de manera semestral.

Palabras clave: Situs Inversus; dextrocardia; síndrome de preexcitación.

\section{Situs inversus totalis and Pre Excitation Syndrome}

Situs inversus totalis is the complete congenital inversion of thoracic and abdominal organs. We present the case of a 3-year-old girl with no previous medical history. When seen with an acute respiratory syndrome, dextrocardia and gastric bubble on the right side led to the diagnosis of Situs inversus. She was referred to infant cardiology where the diagnosis was confirmed with a transthoracic echocardiogram.
In addition, the electrocardiogram identified the presence of ventricular preexitation. Once the acute respiratory symptoms subsided, the patient remains controlled periodically in primary care and by a specialist every six months. No episodes of tachycardia have been detected

Keywords: Situs inversus; dextrocardia, preexcitation syndrome. 


\section{Introducción:}

El término "situs" deriva del latín y se define como el lugar donde existe o se origina algo. En medicina, se refiere principalmente a la posición de los órganos no pareados, como el corazón, bazo, hígado y el estómago en relación a la línea media ${ }^{1}$. Usando la clasificación de Corral et al (basada en la terminología de Fulcher y Turner ${ }^{2}$ el Situs Solitus se refiere a la posición normal a izquierda del ápex cardíaco, el bazo y estómago; a derecha el hígado, la vesícula biliar y la vena cava; y la correcta posición del colon y las asas de intestino delgado. El Situs Inversus hace referencia de una configuración en espejo con dos categorías: situs inversus con dextro o levocardia ${ }^{3}$. La primera de estas es más frecuente y se nomina Situs Inversus Totalis (SIT) 4 .

El SIT es una malformación congénita, que fue descrita en 1788 por el patólogo escocés Mathew Baillie ${ }^{5}$. Ha-

bitualmente es asintomática, y presenta una prevalencia estimada de 1/10.000 nacimientos, siendo más frecuente en hombres que mujeres en una relación 1.5:1 y sin predilección racial $^{6}$. Frecuentemente el diagnóstico se realiza de forma casual durante la edad pediátrica dada la ausencia de síntomas, aunque puede llegar a ser descubierto incidentalmente en edad adulta ${ }^{5,7}$.

En el siguiente reporte presentamos un caso de una preescolar que es llevada por su madre a atención primaria (AP) por un cuadro respiratorio agudo donde se pesquisa, de manera incidental, el SIT asociado a síndrome de preexcitación ventricular.

\section{Caso Clínico:}

Paciente de sexo femenino de 3 años, sin antecedentes mórbidos, fue evaluada en atención primaria por un cuadro de 3 días de evolución de tos con expectoración

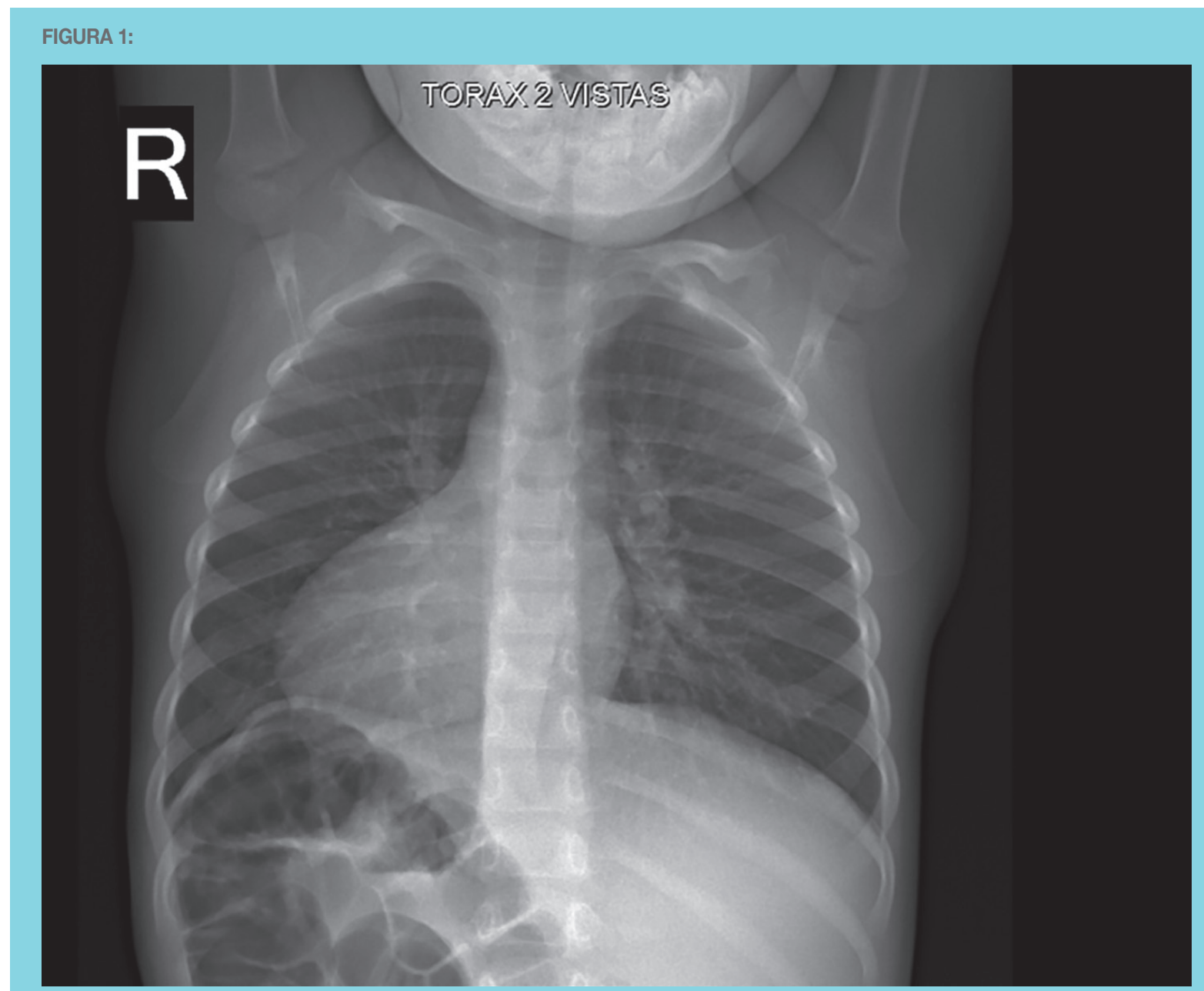

Radiografía de tórax AP. Se observa el Situs inversus totalis, con dextrocardia, la cámara gástrica con contenido aéreo en la región infradiafragmática derecha y la sombra hepática en la región infradiafragmática izquierda. 
mucosa, sin otros síntomas asociados. Al examen físico se auscultaron sibilancias difusas y crépitos en la base izquierda, por lo que se solicitó una radiografía de tórax, iniciándose tratamiento antibiótico empírico, broncodilatadores y kinesioterapia.

Se controló a las 24 horas con la radiografía de tórax, donde se evidenció dextrocardia, burbuja gástrica a derecha y sombra hepática a izquierda. Al examen físico dirigido se auscultaron ruidos cardíacos a derecha, ritmo en dos tiempos sin soplos y matidez hepática a izquierda, con palpación de borde hepático a nivel de reborde costal izquierdo. Tras resolver cuadro agudo se derivó a cardiología infantil donde se estudia con ecocardiograma transtorácico, que confirma el situs inversus totalis, dextrocardia asociada a insuficiencia mitral leve-moderada, ventrículo izquierdo con disfunción leve por alteración del movimiento septal alto e hipertrabeculación del ápex. Al electrocardiograma (ECG) se describe un ritmo sinusal, P negativa en DI, intervalo PR corto, onda Delta y QRS ancho, eje en $-10^{\circ}$, precordiales con predominio de ondas negativas a izquierda, por lo que se diagnosticó dextrocardia asociado a un síndrome de preexcitación.

Se educó sobre condición a los familiares evitando el uso de cardio estimulantes, control periódico con odontólogo para eventual necesidad de profilaxis de endocarditis; y se inició enalapril con control de especialidad a los 6 meses.

\section{Discusión}

Se estima que un 99,99\% de los seres humanos desarrollan durante la etapa embrionaria una asimetría izquierda-derecha de los órganos toracoabdominales, descrita previamente como situs solitus ${ }^{8}$. El SIT se caracteriza por la posición invertida de los órganos con respecto al plano sagital como resultado de un defecto en la tercera semana del desarrollo embriológico, etapa en la que establecen los ejes craneocaudal, dorsoventral y derecha-izquierda del embrión ${ }^{7,9}$. Aunque la patogenia no está bien definida, se cree que se hereda de forma autosómica recesiva $^{5}$, pudiéndose encontrar en gemelos idénticos ${ }^{10}$.

Al ser asintomática, generalmente la sospecha inicial es un hallazgo casual a la exploración física o radiografía de tórax. Posteriormente, es necesario completar el estudio con imágenes más específicas como el ultrasonido, tomografía computarizada o resonancia magnética con el fin de estudiar posible anomalías estructurales ${ }^{6,11}$. El ecocardiograma es importante para establecer el situs, las relaciones auriculoventriculares y ventriculoarteriales, definir los drenajes sistémicos y pulmonares y descar- tar una patología cardíaca estructural. También se puede agregar una ecografía abdominal para detectar posibles malformaciones como hernia diafragmática, asplenia o poliesplenia $^{12}$. En nuestra paciente, el SIT se sospechó con el resultado de la radiografía de tórax inicial, ya que solo se realizó un examen pulmonar, omitiendo la revisión de otros sistemas. Esto podría haber despertado la sospecha.

Ya sea como hallazgo de rutina o como parte del estudio, es necesario conocer las posibles alteraciones que pueden encontrarse en el ECG realizado de manera clásica: onda P negativa en la derivación DI, negativización del QRS y de la onda T en DI, eje eléctrico a derecha y poca progresión de onda R en precordiales. En estos caso es necesario colocar los electrodos de los miembros superiores de manera invertida, manteniendo igual los de los miembros inferiores. Los electrodos precordiales se colocarán en "espejo" a la forma tradicional ${ }^{13}$.

La presencia de cardiopatía congénita (CC) en población normal, es decir, que presentan Situs Solitus es del 1\%, mientras que en el SIT varia de un 3-5\%. Su variante con levocardia presenta $\mathrm{CC}$ en prácticamente todos los ca$\operatorname{sos}^{2,12}$. Dentro de las CC se pueden encontrar la transposición de grandes vasos, estenosis pulmonar, comunicación interauricular e interventricular y retorno venoso anómalo ${ }^{6}$. Se estima que la prevalencia del síndrome de preexcitación en población general es de 0.68 a 1.7 en 1000, siendo 0.08 en 1000 en el subgrupo de 1 a 4 años de edad ${ }^{14}$. Hay pocos reportes que estimen la prevalencia en población con $\mathrm{CC}$, pero creemos que debería aumentar considerablemente.

El SIT se puede asociar a otras anomalías congénitas como atresia duodenal, asplenia, poliesplenia, riñones ectópicos, riñón en herradura, alteraciones vasculares y pulmonares y patologías malignas gastrointestinales 6,11 . Además, cuando el SIT se asocia a disquinesia ciliar primaria se denomina como síndrome de Kartagener ${ }^{15}$.

En general, los pacientes con SIT sin anomalías congénitas pueden disfrutar de vidas normales con una expectativa de vida similar a su cohorte según edad y sexo ${ }^{16}$. Es importante el diagnóstico precoz y la educación de los familiares ya que las enfermedades agudas, como la apendicitis, colecistitis o infarto agudo de miocardio pueden presentarse de forma atípica y ser confundidos con cuadros benignos, retrasando posibles tratamientos o cirugías de urgencia 7 .

\section{Conclusión}

El SIT es una entidad poco frecuente, pero que puede 
estar asociado a otras comorbilidades, en especial cardiopatía congénita. Es importante realizar siempre un adecuado examen físico, en especial en la edad pediátrica para poder identificar posible casos y evitar realizar exámenes innecesarios ante hallazgos incorrectos como al tomar un ECG con electrodos en posiciones clásicas. A pesar de que la mayoría de los casos de SIT tendrán una vida normal, se debe educar a los pacientes y/o familiares sobre la condición y las posibles presentaciones atípicas de patologías agudas.

\section{Bibliografía}

1. DRISCOLL DJ, Presentation and therapy of defects of situs, Congenital Heart Diseases: The Broken Heart, Vienna, Springer, 2016, 461-462.

2. FULCHER AS, TURNER MA. Abdominal manifestations of situs anomalies in adults. Radiographics. 2002; 22(6): 1439-1456.

3. CORRAL G, LABRA A, SCHIAPPACASSE G. Manifestaciones abdominales de las anomalías del Situs Ambiguous en el adulto: A propósito de cuatro casos. Rev Chil Cardiol. 2013; 19(1): 38-43.

4. MONCAYO RA, GRANIZO L, COBO H. Reporte de Caso: Situs Inversus Totalis. Rev Cuban Cardiol. 2019; 24(4): 438-443.

5. TABRY I, et al. Case report: off-pump total myocardial revascularization for dextrocardia and situs inversus. Heart Surg Foru. 2001; 4(3): 251-253.

6. REALES V, GALLEGO G, ESPITIA N, COLEY A, SUAREZ O. Situs inversus totalis: revisión de tema con aproximación a la Genética y reporte de casos. Rev Col Cardiol. 2017; 24(1): 40-47.

7. KHALIL S, KAMLA A, AHMED S. Acute myocardial infarction in a patient with dextrocardia and situs inversus. J Electrocardiol. 2005; 38(4): 412-413.

8. AYLSWORTH A. Clinical aspects of defects in the determination of laterality. AJMG. 2001; 101(4): 345-355.
9. CARRILLO E, et al. (2012). Situs inversus totalis. Med Int Mex. 2012; 28(2): 187-191.

10. BHANDIWAD A, GOWDA S. Situs Inversus Totalis-A Case Report and Review. JMSCR. 2014: 3(9); 7686-7688

11. PEREZ M, et al. Kartagener syndrome: neonatal diagnosis. A case report. Arch Argent Pediatr. 2019; 117(3). 292-296.

12. JUNCOS M, ROS M, MARAVALL M, ALVAREZ-PITTI J. Situs inversus totalis: A propósito de 2 casos clínicos. Rev Chil Pediatr. 2014; 85(3): 344-350.

13. RAMOS T, TRAPERO J, SANTAREN D. Reconocimiento electrocardiográfico de una dextrocardia. Aten Primaria. 2017; 49(2): 120.

14. Lu C, WU M, CHEN H, KAO F, HUANG S. (2014). Epidemiological profile of Wolff-Parkinson-White syndrome in a general population younger than 50 years of age in an era of radiofrequency catheter ablation. Int J Cardiol. 2014; 174(3): 530-534.

15. YILMAZ S, DEMIRTAS A, TOKPINAR A, ACER N.. Dextrocardia y Situs Inversus Totalis en un Sujeto Turco: Reporte de un Caso. Int J Morphol. 2019; 37(3): 900-902.

16. BOHUN C, POTTS J, CASEY B, SANDOR G. (2007). A population-based study of cardiac malformations and outcomes associated with dextrocardia. Am J Cardiol. 2007; 100(2); 305-309. 